Schweiz. Z. Tuberk. 1955;12:1

\title{
Auguste Rollier 1874 à 1954
}

La carrière à-Auguste Rol-lier, né le ler octobre 1874 et mort à Leysin le 30 octobre 1954, marque une page importante dans Thistoire de nos conceptions de la maladie tuberculeuse et de son traitement.

Sa biographie a été écrite ailleurs. Nous ne vou-lons pas en rappeler une fois de plus les dates, mais lui rendre un hommage recon-naissant en rappelant les trois domaines dans lesquels il a été un novateur et un créateur.

Au debut du XXe siècle, à un moment où les progrès spectaculaires de la chirurgie opératoire avaient permis un instant de croire qu'elle allait guérir des tuberculeux par le bistouri, Rollier a poussé un cri d'alarme en rappelant que la tuberculose est une maladie générale et qu'il fallait avant tout traiter le malade entier pour le guérir de sa maladie. Par une inlassable patience qu'il a su faire partager à ses malades, associée à une imagination technique et orthopédique fertile, il a su obtenir du traitement conservateur, applique dans des conditions hygiéniques et climatiques idéales, les résultats les meilleurs et les plus durables.

En faisant bénéficier au maximum ses malades des vertus curatrices du grand air et de la lumière solaire, il a realise le role eminent que ces deux elements exercent sur la santé générale. II a contribué à vulgariser Гédu-cation en plein air et à la lumière, facteur prophylactique de santé. Le troisíème domaine dans lequel il a été un créateur et un novateur, c'est en faisant de la médecine «psycho-somatique» avant la lettre. II n'a cessé de se préoccuper de la santé morale de ses malades, de la nécessité d'occuper sainement ses fonctions intellectuelles et psychiques pendant Гimmobilisation prolongée nécessitée par le traitement; de le préparer tout au long de ce traitement à son retour dans sa famille, dans sa vie professionnelle et dans la société.

Dans ces trois domaines, Rollier a été un précurseur et a vu juste

d'emblée. Rien n'est venu infirmer l'exactitude de ces principes. Bien plus, on n'a cessé d'en étendre les applications dans la suite et jusqu'à aujourd'hui. A ce titre, Rollier restera une figure marquante dans $\Gamma$ histoire non seulement de nos conceptions de la maladie tuberculeuse, mais de la maladie et de Thygiène en general. P. Steiner et M. Wasserfallen, Leysin 